\title{
Ethnobotanical use of Stachys L. (Lamiaceae) taxa in Turkey
}

\author{
Fatih Satıl'1, Mikail Açar² \\ ${ }^{1}$ Balıkesir University, Faculty of Arts and Sciences, Department of Biology, Balıkesir, Turkey, \\ orcid.org/0000-0002-4938-1161 \\ 2Munzur University, Tunceli Vocational School, Department of Plant and Animal Production, Tunceli, Turkey, \\ orcid.org/0000-0003-3848-5798 \\ *Corresponding author: mikailacar27@hotmail.com
}

Received: 12 May 2020, Revised: 16 June 2020, Published Online: 01 December 2020

\begin{abstract}
The purpose of this study was to determine the ethnobotanical features of taxa belonging to the genus Stachys $L$. in Turkish Flora. Their vernacular names and local usages patterns have been determined. To determine the ethnobotanical uses of Stachys L. taxa; ethnobotanical studies made in Turkey have examined. Also, the field study carried out in different regions in Turkey. The ethnobotanical use of 38 Stachys L. taxa (29 species) has been identified in Turkey. The first 2 species that are used most: S. lavandulifolia and S. cretica. They are used 59 different of the vernacular names for Stachys L. taxa in Turkey, They are mostly known in Anatolia as "Dağ çayı". Also "Bareş", "Çaye çe", 'Rıhena tehtan' and "Tokalı çay" are among the names given. Stachys L. species are generally consumed as a herbal tea for medicinal in Turkey. Besides, they are used for as powder for animal disease, gargle for sore throat and handkerchief and hair accessories (both from leaves) for children. In terms of food as a spice, only the $S$. mardinensis species was observed. Stachys $L$. taxa have been revealed that they are being used in the treatment of about 40 different diseases and symptoms. Top diseases and symptoms treated with Stachys L. taxa: stomathic, cold, cough and diabetes. The most used parts of the plants are listed aerial parts and leaves.
\end{abstract}

Key words: Ethnobotany, folk medicine, herbal tea, Lamiaceae, medicinal plant, Stachys L.

\section{Introduction}

Etnobotany is defined simply as "the study of the relationships between plants and people". Documentation of the indigenous knowledge through ethnobotanical studies important for the conservation and utilization of biological resources (Muthu et al., 2006). Therefore, the establishment of the local names and indigenous uses of plants has significant potential societal benefits (Bağcl, 2000). 
Several studies have been published recently on the ethnobotany of Turkey (Sezik et al., 1991; İlçim and Varol, 1996; Sezik et al., 1997; Özgen et al., 2004; Ezer and Avcl, 2004; Everest, and Öztürk, 2005; Elçi and Erik, 2006; Ezer and Arısan, 2006). Furthermore, Ertuğ (2000) and Baytop (1984, 1994) published intensive researches provided considerable information not only on medicinal plants but also on edible plants, fodder, fuel, dyes and gums. Many more detailed studies are necessary to obtain a comprehensive picture of plant-human interactions in Turkey.

Turkey is regarded as an important centre of biodiversity for the Lamiaceae. In Turkey, the family is represented by 603 species and a total of 782 taxa (346 endemics) (Celep and Dirmenci, 2017) The extracts and essential oils of some of the species from Lamiaceae family have been used as traditional medicine for some diseases, as a food source and food preservative for thousands of years. Stachys L. species are one of those species. The genus Stachys L., one of the largest genera of the Lamiaceae (Labiatae) family. Stachys $L$. is a taxonomically large and complex genus of Lamiaceae family. While it is a subcosmopolitan genus, it spreads in the Mediterranean and Southwest Asia, secondly in North and South America and North Africa, but not in Australia and New Zealand (Bhattacharjee, 1980). It is represented by approximately 370 species and 435 taxa in the world (Harley et al., 2004; Govaerts, 2003). In Turkey, there are 91 species and 118 taxa of the genus Stachys L. 57 of these taxa are endemic and the rate of endemism is 48\% (Davis, 1982; Guner et al., 2000; Akçiçek et al., 2016). Stachys L. is a Greek word, meaning "ear of corn" or "spike" and refers to the arrangement of flowers on the stem (Kaya et al., 2001). They are consumed as herbal remedies in alternative medicine and wild tea (mountain tea) in Mediterranean regions. There are many chemical studies on the essential oils of Stachys $L$. taxa. The essential oil composition of the species is one of the main reasons for their consumption as tea in Anatolian ethnobotany (Altundag and Öztürk, 2011; Polat et al., 2012); however, the species also consist of glycosides, saponins, polyphenols, tannins, phenolic acids, flavonoids, and diterpenoids together with essential oils, mono and sesquiterpenoids. Therefore, the synergistic effects of the component chemicals could be the main reason of consumption of their flowers and aerial parts as a tea for medicinal purposes in Anatolian culture (Dönmez et al., 2012; Goren, 2014; Kaya et al., 2017,). Some members of the genus have been reported to be used as anti-inflammatory and antibacterial agents. Moreover, their antianxiety, antioxidant and antinephritic properties have also been reported (Hayashi et al., 1994; Takeda et al., 1996; Maleki et al., 2001; Goren et al., 2011a, b).

Several studies and ethnobotanical notes are available indicating the consumption of some Stachys L. taxa in Anatolian culture. But, a specific ethnobotanical study has not been found only on the Stachys L. genus. In this review, ethnobotanical properties of the Stachys L. genus which spread in different regions of Turkey and their consumption as food and herbal remedies will be discussed. 


\section{Material and Methods}

To determine the ethnobotanical uses of taxa determined during the field studies; plants were shown to local people and their ethnobotanical uses were revealed (Figure 1). Ethnobotanical studies were carried out in 13 provinces (Adıyaman, Balıkesir, Bursa, Çanakkale, Denizli, İznik, Kahramanmaraş, Kütahya, Manisa, Mardin, Şanlıurfa, Tunceli, Van). A total of 98 informants, ages 20 and over were reached. In addition, herbal markets and bazaars were also visited. During the study, face-to-face interviews made with the informants were determined local names and usage of taxa. Also the ethnobotanical uses of Stachys L. taxa ethnobotanical studies (article and thesis) made in Turkey have examined. In addition, essential oil studies and biological activity studies on the Stachys L. have been scanned. In these studies, the ethnobotanical uses and local names of Stachys L. taxa were comprehensively recorded (Table 1). Turkish names of plants are given according to Güner et al. (2012). National Thesis Center database has been used for theses in the study.

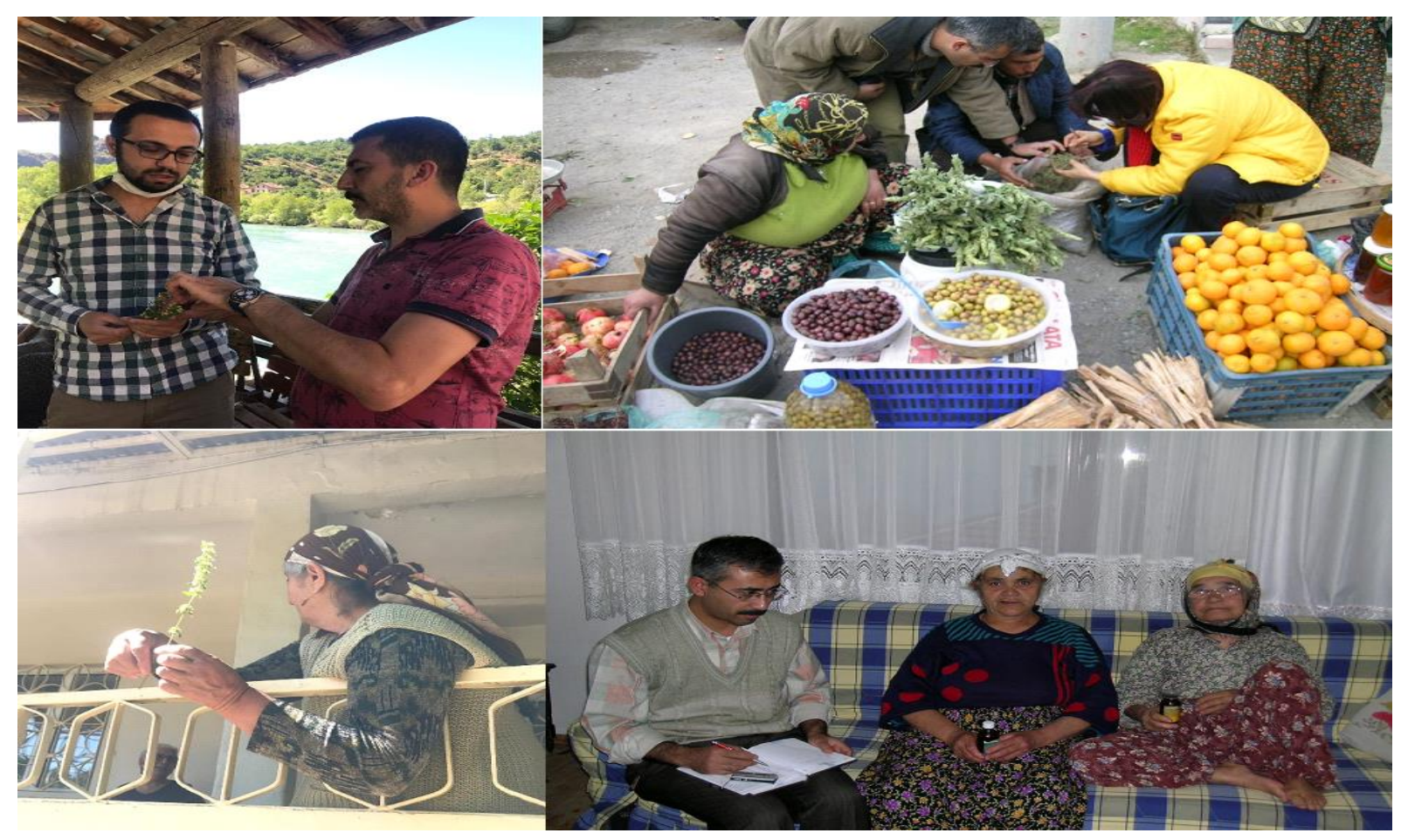

Figure 1. Field studies on genus Stachys $L$.

\section{Results and Discussion}

The ethnobotanical use of 38 Stachys L. taxa (29 species) has been identified in Turkey (Table 2). The first 2 species that are used most: S. lavandulifolia Vahl and S. cretica L. (Figure 3). 
Stachys $L$. taxa spreading different regions of Turkey is referred to by many different vernacular names (Table 1). They are used 59 different names for Stachys $L$. taxa in Turkey.

They are mostly known as "Dağ çayı" in Anatolia. In addition 'Bareş', 'Çaye çe', 'Rıhena tehtan' and 'Tokalı çay' are among the names given (Figure 5; Table 1). Other names, except for mountain tea (Dağ çayı), are not commonly used names. They are just similar names given to some taxa.

Stachys L. taxa have been revealed that they are used in the treatment of about 40 different diseases and symptoms. Top diseases treated with Stachys L. taxa: Stomathic, cold, cough and diabetes (Figure 7, Table 1).

According to ethnobotanical studies and the literature survey, Stachys L. species are used widely as herbal tea particularly in the region covering from East to West Anatolia (Figure 2).

It was observed that the province with the most usage records was Şanlıurfa, Balıkesir, Bilecik and Rize. In addition, Thrace (such as Edirne, Tekirdağ, Kırklareli), Eastern Anatolia (such as Iğdır, Ağrı, Kars) and Southeastern Anatolia (such as Siirt, Hakkâri, Şırnak) and the western parts of the Aegean (such as Muğla, Aydın, İzmir). It was observed that they were not used. However, Stachys L. species are found in Turkey's in almost every region. It may be thought that the reason for their lack of these uses is due to the limited number of ethnobotanical studies.

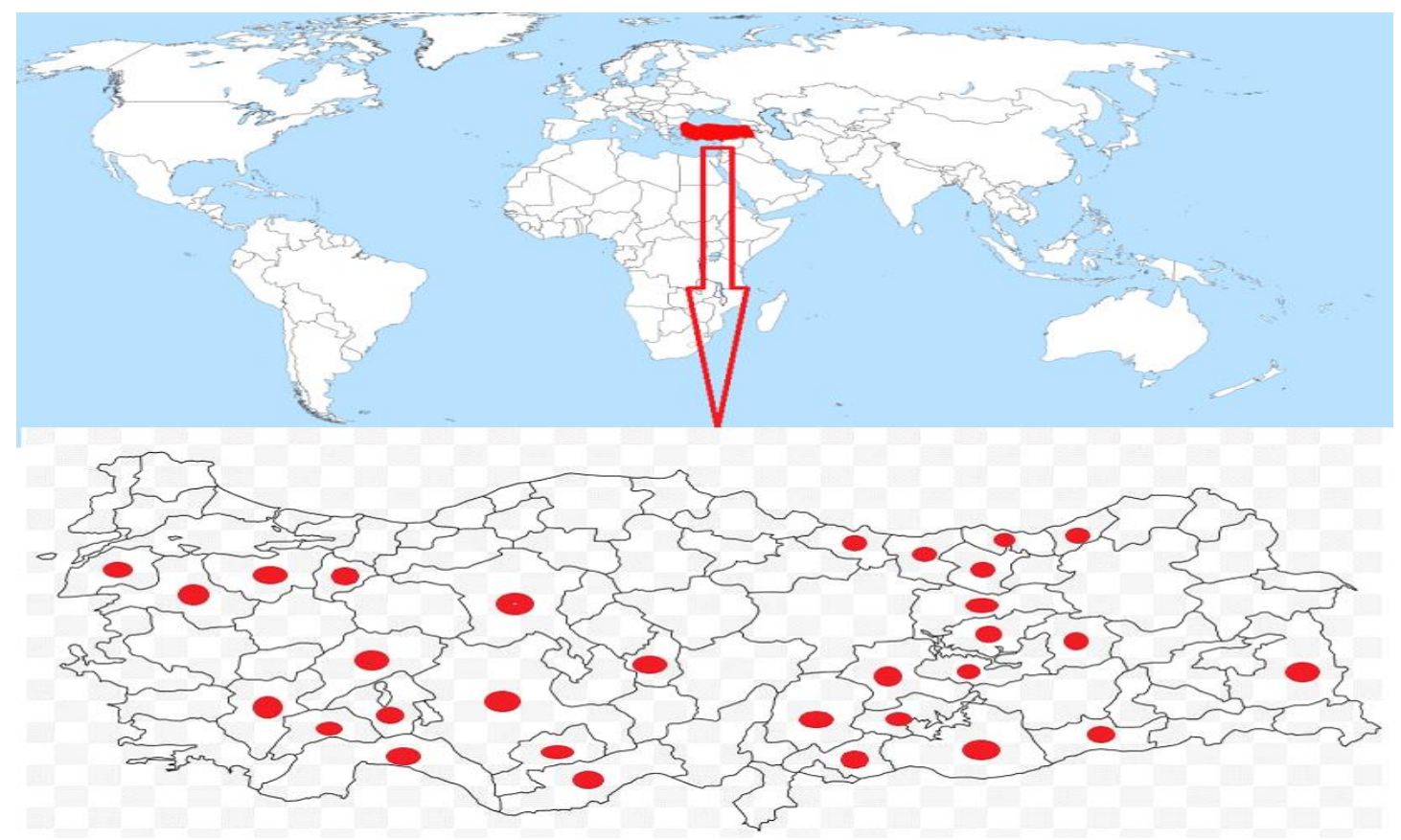

Figure 2. The map showing the provinces with ethnobotanical use of Stachys L. taxa in Turkey. 


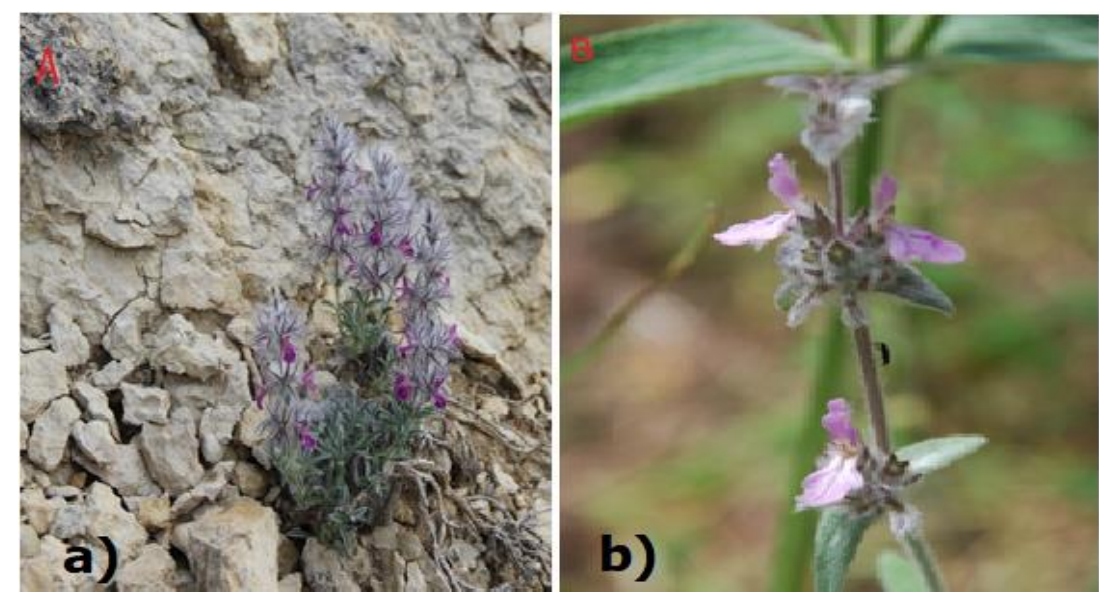

Figure 3. The most used species a) S. lavandulifolia b) S. cretica (Photo: Satı F, Selvi S).

Stachys $L$. taxa are generally consumed as a herbal tea for medicinal in Turkey. In addition, in rare cases, in powder form in animal diseases (S. balansae var. balansae Boiss. \& Kotschy, S. kurdica var. kurdica Boiss. \& Hohen., S. lavandulifolia var. lavandulifolia Vahl) in the form of application to wounds (S. cretica subsp. smyrnaea Rech.f.), in the form of gargling in throat disorders (S. lavandulifolia var. glabrescens R.Bhattacharjee \& Hub.-Mor.) has been observed in some places, such as handkerchief and hair accessories (S. cretica L., S. byzantina K.Koch) for children, as well as in some places such as aroma and spice (S. mardinensis (Post) R.R. Mill).

The most used parts of the plants are listed aerial parts (\%52) and leaves (\%20) (Figure 6, Table 1).

In our country, S. cretica is represented by 12 taxa. The S. cretica taxa used in our country are as follows: S. cretica subsp. anatolica Rech.f., S. cretica subsp. garana (Boiss.) Rech.f., S. cretica subsp. lesbiaca Rech.f., S. cretica subsp. mersinaea (Boiss.) Rech.f., S. cretica subsp. mersinaea (Boiss.) Rech.f., S. cretica subsp. smyrnaea Rech.f.

S. lavandulifolia is used in such as stimulant energizer antipyretic stomachic. Since $S$. lavandulifolia is used in many regions of Anatolia, it can be used for many purposes (Table 1).

Different subcategories of the same species can be used in different diseases. For example $S$. cretica subsp. anatolica Rech.f. is effective in rabies treatment for animals despite that $S$. cretica subsp. garana (Boiss.) Rech.f. for the treatment of Alzheimer disease.

Generally, infusion or decoction (as tea) form many of the species have been reported in the literature (Table 1).

The leaves and aerial parts of $S$. mardinensis are consumed against headache, diabetes treatment, cough, bronchitis and it is also aromatic for local food in Southeastern Anatolia. In terms of food as a spice, only the $S$. mardinensis species was observed. 
S. iberica M.Bieb. is used for the treatment of wounds. In addition to tea, some of the Stachys $L$. taxa are used in some cases as a powder. This method of use is generally used in animal diseases ( $S$. balansae var. balansae, S. kurdica var. kurdica, S. lavandulifolia var. lavandulifolia). The fresh aerial parts of these plants are dried and poured into powder. Then it is used to treat the inflamed wounds of for livestock. This method of use is specified by the same source person.

In addition, except medical purpose, some species provide use for children. In the literature, it was observed that only two of the Stachys $L$. species were used for the purpose of hair accessories and handkerchiefs (S. cretica, S. byzantina). Endemic S. cretica subsp. smyrnaea has been demonstrated that is used only in certain regions (Aegean). This herb is used for wounds and stomachic (Figure 4).

It has also been reported that there are side effects in the use of some taxa. These $S$. lavandulifolia var. lavandulifolia (Tea increases blood pressure) and S. lavandulifolia var. glabrescens (Increases blood pressure if consumed more). In addition, it was observed that $S$. lavandulifolia was named with different vernacular names. The vernacular name of the plant is 'Seyitrıza çayı' (Tunceli) and 'Reyyan' (Van) in the East Anatolian Region.

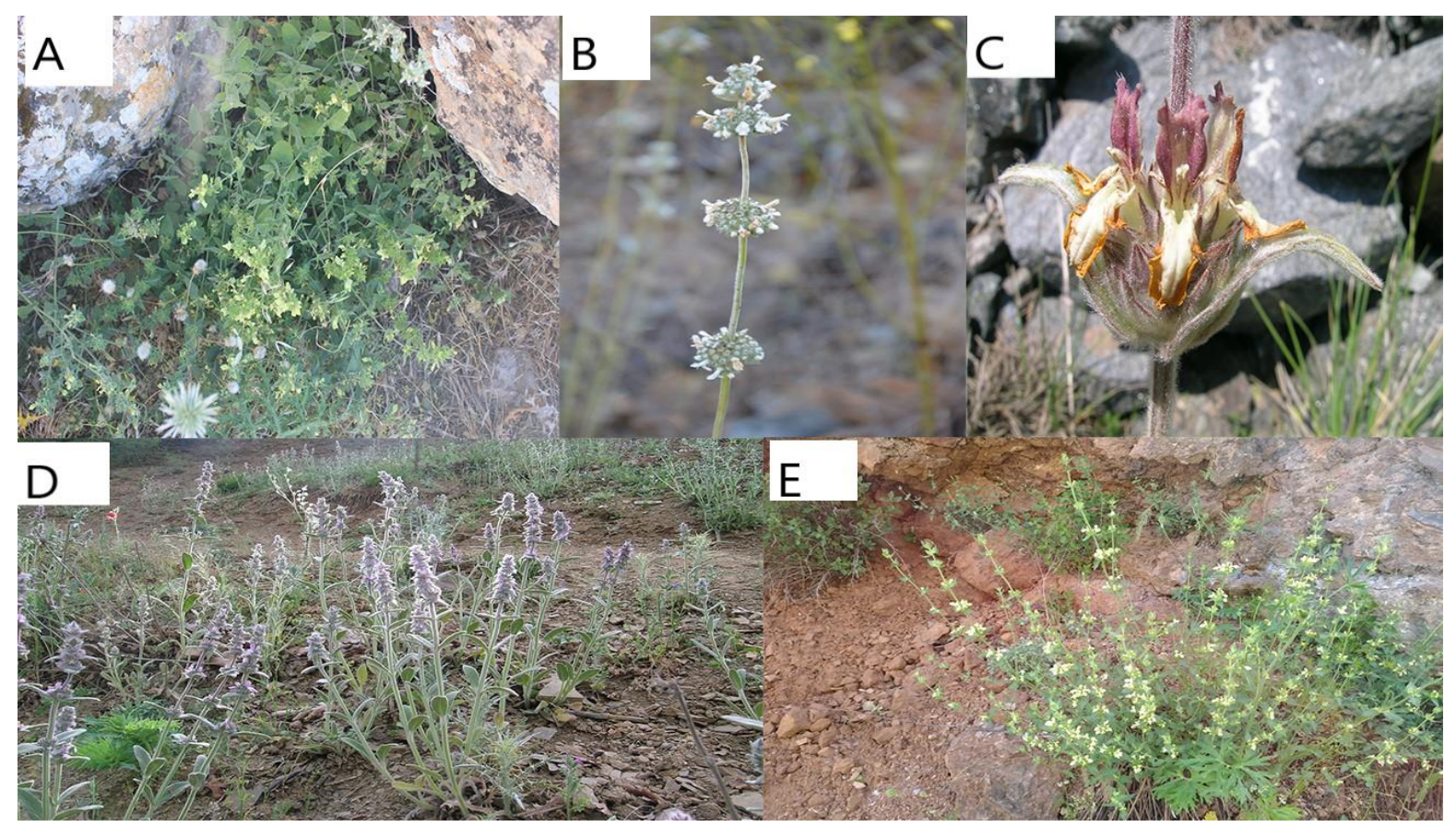

Figure 4. Some Stachys L. taxa with ethnobotanical use A) S. mardinensis, B) S. tmolea, C) S. cretica subsp. smyrnea, D) S. byzantina, E) S. viscosa. 
Table 1. Ethnobotanical features based on the literature of Stachys $L$. taxa in Turkey.

\begin{tabular}{|c|c|c|c|c|c|c|c|}
\hline Taxa & Turkish name & Vernacular name & Region/Province & Parts of use & Usage & Purpose of usage & References \\
\hline $\begin{array}{l}\text { S. aleurites Boiss. \& } \\
\text { Heldr.* }\end{array}$ & Köprülüçay & Tokalı çay & $\begin{array}{l}\text { Western } \\
\text { Mediterranean } \\
\text { region (Burdur, } \\
\text { Isparta, Antalya) } \\
\end{array}$ & $\begin{array}{l}\text { Aerial parts, } \\
\text { inflorescence, } \\
\text { leaves }\end{array}$ & Tea (infusion) & $\begin{array}{l}\text { Cold and flu, stomach ache, anodyne, } \\
\text { appetizing }\end{array}$ & Fakir et al., 2009 \\
\hline $\begin{array}{l}\text { S. annua subsp. } \\
\text { annua var. annua (L.) } \\
\text { L. }\end{array}$ & Hacıosmanotu & Dağ çayı & Bilecik & $\begin{array}{l}\text { Leaves and aerial } \\
\text { parts }\end{array}$ & Tea & Insomnia, menstrual disorders & $\begin{array}{l}\text { Koyuncu et al., 2010; } \\
\text { Tuzlacı, } 2011 \text { (As cited } \\
\text { in Ertuğ, 2014) }\end{array}$ \\
\hline $\begin{array}{l}\text { S. annua subsp. } \\
\text { annua var. lycaonica } \\
\text { R.Bhattacharjee }\end{array}$ & & $\begin{array}{l}\text { Dağ çayı, } \\
\text { Hacıosman otu }\end{array}$ & $\begin{array}{l}\text { East Anatolia, } \\
\text { Bilecik, Nevşehir, } \\
\text { Tunceli }\end{array}$ & Aerial parts & $\begin{array}{l}\text { Tea (infusion and } \\
\text { decoction/internal) }\end{array}$ & $\begin{array}{l}\text { Insomnia, menstrual disorders colds, } \\
\text { antipyretic, expectorant, rheumatism, } \\
\text { lowering cholesterol, diabetes (lowering } \\
\text { blood sugar) }\end{array}$ & $\begin{array}{l}\text { Koyuncu et al., 2010; } \\
\text { Altundag and Ozturk, } \\
\text { 2011; Şenkardeş, } 2014\end{array}$ \\
\hline S. arvensis (L.) L. & Tarlakarabaşı & Mayasıl otu & Rize & Aerial parts & Tea (Decoction/internal) & Hemorrhoids & Saraç et al., 2013 \\
\hline $\begin{array}{l}\text { S. balansae var. } \\
\text { balansae Boiss. \& } \\
\text { Kotschy* }\end{array}$ & Bozçayçe & Bareş & Van & Aerial parts & Powder & Animal disease (for livestock) & Mükemre, 2013 \\
\hline S. brantii Benth. ${ }^{*}$ & Yitikçayçe & \multicolumn{4}{|c|}{ Original source is not specified. No usage details are given in the source. } & Medicinal & Koуu, 2020 \\
\hline $\begin{array}{l}\text { S. burgsdorffioides } \\
\text { subsp. ladanoides } \\
\text { Hand.-Mazz. }{ }^{*}\end{array}$ & Eğinkarabaşı & Eğin karabaşı & Şanlıurfa & & Mix with thyme & Hemorrhoids & Şeker, 2018 \\
\hline S. byzantina K.Koch & Bozkarabaş & Eşek otu & $\begin{array}{l}\text { Bilecik, Erzincan, } \\
\text { Ordu, Balıkesir }\end{array}$ & $\begin{array}{l}\text { Aerial parts, } \\
\text { inflorescence, } \\
\text { leaves }\end{array}$ & $\begin{array}{l}\text { Tea, handkerchief } \\
\text { (leaves), nectar for bees }\end{array}$ & Colds, forage & $\begin{array}{l}\text { Koyuncu et al., 2010; } \\
\text { Alpaslan, 2012; } \\
\text { Badem, } 2017\end{array}$ \\
\hline S. cretica L. & Deliçay & $\begin{array}{l}\text { Dağ çayı, Şalba } \\
\text { çayı, Pamuk } \\
\text { prenses, Dut } \\
\text { çiçeği }\end{array}$ & $\begin{array}{l}\text { Balıkesir, Konya, } \\
\text { Afyon, Mersin, Rize }\end{array}$ & $\begin{array}{l}\text { Flowering } \\
\text { branches, aerial } \\
\text { parts, leaves }\end{array}$ & $\begin{array}{l}\text { Tea (infusion), hair } \\
\text { accessories, food } \\
\text { (nectar) }\end{array}$ & $\begin{array}{l}\text { Stomach ache, stimulant, dsypnea } \\
\text { healing, anodyne, tonic, wound healing, } \\
\text { food }\end{array}$ & $\begin{array}{l}\text { Yeşilada et al., 1995; } \\
\text { lşık et al., 1995; Metin, } \\
\text { 2009; Polat and Satıl } \\
\text { 2012; Baykal, } 2015\end{array}$ \\
\hline $\begin{array}{l}\text { S. cretica subsp. } \\
\text { anatolica Rech.f. }\end{array}$ & Yağlıkara & $\begin{array}{l}\text { Çaya çe, Dağ } \\
\text { çayı, Çay otu, } \\
\text { Kestire, Ballık, } \\
\text { Oğul otu, Boz } \\
\text { çalba, Beyaz } \\
\text { şabla, Karabaş } \\
\text { otu }\end{array}$ & $\begin{array}{l}\text { East Anatolia, } \\
\text { Malatya, Konya, } \\
\text { Bilecik, Denizli, } \\
\text { Ankara, Balıkesir }\end{array}$ & $\begin{array}{l}\text { Aerial part, } \\
\text { leaves, } \\
\text { inflorescence }\end{array}$ & $\begin{array}{l}\text { Tea (infusion, decoction } \\
\text { linternal), honey plant } \\
\text { for bees }\end{array}$ & $\begin{array}{l}\text { Colds and stomach ailments, forage, } \\
\text { respiratory tract diseases, stomachic, } \\
\text { anadoyne, rabies treatment (drink to } \\
\text { animals) }\end{array}$ & $\begin{array}{l}\text { Ertuğ et al., 2004; } \\
\text { Yeşil, 2007; Vural, } \\
\text { 2008; Yeşil \& Akalın, } \\
\text { 2009; Koyuncu et al., } \\
\text { 2010; Ayandın, 2010; } \\
\text { Keskin, 2011; Altundag } \\
\text { and Ozturk, 2011; } \\
\text { Özdemir, 2016 }\end{array}$ \\
\hline $\begin{array}{l}\text { S. cretica subsp. } \\
\text { garana (Boiss.) } \\
\text { Rech.f. }\end{array}$ & Kabaçay & Kabaçay & Şanlıurfa & & Tea & Alzheimer disease & Şeker, 2018 \\
\hline $\begin{array}{l}\text { S. cretica subsp. } \\
\text { lesbiaca Rech.f. }\end{array}$ & Şabıla & $\begin{array}{l}\text { Deli ada çayı, } \\
\text { Şabılla }\end{array}$ & Çanakkale, Denizli & Aerial parts & Tea (infusion internally) & Stomach ache, honey plant (for bees) & $\begin{array}{l}\text { Ertuğ et al., 2004; } \\
\text { Bulut, } 2008\end{array}$ \\
\hline S. cretica subsp. & Boncukşalba & Çaya çe, Dağ & East Anatolia, & Aerial part & Tea (infusion and & Colds and stomach ailments, & Yeşil, 2007; Yeşil and \\
\hline
\end{tabular}




\begin{tabular}{|c|c|c|c|c|c|c|c|}
\hline $\begin{array}{l}\text { mersinaea (Boiss.) } \\
\text { Rech.f. }^{*}\end{array}$ & & çayı, & Malatya & & decoction internally) & & $\begin{array}{l}\text { Akalın, 2009; Altundag } \\
\text { and Ozturk, } 2011\end{array}$ \\
\hline $\begin{array}{l}\text { S. cretica subsp. } \\
\text { smyrnaea Rech.f. }\end{array}$ & İzmirdeliçayı & Dağ çayı & Balıkesir, Denizli & $\begin{array}{l}\text { Aerial parts root, } \\
\text { leaves }\end{array}$ & $\begin{array}{l}\text { Applied on wounds, } \\
\text { infusion }\end{array}$ & Wounds, stomachic & $\begin{array}{l}\text { Ertuğ et al., 2004; } \\
\text { Güner, 2016; Polat, } \\
2010\end{array}$ \\
\hline $\begin{array}{l}\text { S. gaziantepensis M. } \\
\text { Dinç and S. Doğu }\end{array}$ & & & Gaziantep & & Tea & Cold & Kaya et al., 2017 \\
\hline S. iberica M.Bieb. & Tokdeliçay & Dağçayı & Afyon, Nevşehir & Leaves & & $\begin{array}{l}\text { Stomachic, stimulant, dsypnea healing } \\
\text { (good for shortness of breath), anodyne, } \\
\text { tonic and wound healing }\end{array}$ & $\begin{array}{l}\text { Öztürk and Özçelik, } \\
1991 \text { (as cited in Ertuğ, } \\
\text { 2014); Işık et al., 1995; }\end{array}$ \\
\hline $\begin{array}{l}\text { S. iberica subsp. } \\
\text { georgica Rech.f. }\end{array}$ & Üçdeliçay & Dağ çayı & East Anatolia & Aerial parts & Tea (decoction/internal) & Colds, antipyretic & $\begin{array}{l}\text { Altundag and Ozturk } \\
2011\end{array}$ \\
\hline $\begin{array}{l}\text { S. iberica subsp. } \\
\text { iberica M.Bieb. }\end{array}$ & Tokdeliçay & Karaçekme & Şanlıurfa & Aerial parts & Tea & & Balos and Akan 2007 \\
\hline $\begin{array}{l}\text { S. iberica subsp. } \\
\text { iberica var. iberica } \\
\text { M.Bieb. }\end{array}$ & Tokdeliçay & Tok deliçay & Şanlıurfa & & Tea & Alzheimer disease & Şeker, 2018 \\
\hline $\begin{array}{l}\text { S. iberica subsp. } \\
\text { stenostachya (Boiss.) } \\
\text { Rech.f. }\end{array}$ & Benlideliçay & $\begin{array}{l}\text { Benli çay, Dağ } \\
\text { çayı }\end{array}$ & $\begin{array}{l}\text { East Anatolia, } \\
\text { Şanlıurfa }\end{array}$ & Aerial parts & Tea (decoction/internal) & $\begin{array}{l}\text { Colds, antipyretic, stomach ache, } \\
\text { alzheimer disease }\end{array}$ & $\begin{array}{l}\text { Şeker, 2018; Altundag } \\
\text { and Ozturk, } 2011\end{array}$ \\
\hline $\begin{array}{l}\text { S. kurdica var. kurdica } \\
\text { Boiss. \& Hohen. }\end{array}$ & Karadeliçay & Bareşa kulikzer & Van & $\begin{array}{l}\text { Aerial parts, } \\
\text { flowering } \\
\text { branches }\end{array}$ & Powder, tea & $\begin{array}{l}\text { Animal diseases (for livestock), cold and } \\
\text { stomach ache }\end{array}$ & Mükemre, 2013 \\
\hline S. lavandulifolia Vahl & Tüylü çay & $\begin{array}{l}\text { Dağ çayı, Çay } \\
\text { otu, Tüylü çay, } \\
\text { Bareş, Eşekotu, } \\
\text { Tokalı çay, } \\
\text { Adaçayı, Seyit } \\
\text { Rıza çayı, Çay } \\
\text { otu, Vasse çaye, } \\
\text { Reyyan }\end{array}$ & $\begin{array}{l}\text { East Anatolia, } \\
\text { Konya, } \\
\text { Kahramanmaraş, } \\
\text { Adıyaman, Van, } \\
\text { Antalya, Tunceli }\end{array}$ & $\begin{array}{l}\text { Aerial parts, } \\
\text { inflorescence, } \\
\text { leaves }\end{array}$ & $\begin{array}{l}\text { Tea (decoction, } \\
\text { infusion/internal) }\end{array}$ & $\begin{array}{l}\text { Stimulant, carminative, appetizer, } \\
\text { stomachic, energizer, antipyretic, cough, } \\
\text { food, stomachic, euphoria affect, forage } \\
\text { for livestock (aerial parts) }\end{array}$ & $\begin{array}{l}\text { Öztürk and Özçelik, } \\
\text { 1991; Baytop, } \\
\text { 1994;1999; Özhatay et } \\
\text { al., 1997; Duran, 1998; } \\
\text { Çömlekçioğlu and } \\
\text { Karaman, 2008; } \\
\text { Güldaş, 2009; Altundag } \\
\text { andOzturk, 2011; } \\
\text { Korkmaz and } \\
\text { Demirkuş, } 2019\end{array}$ \\
\hline $\begin{array}{l}\text { S. lavandulifolia var. } \\
\text { lavandulifolia Vahl }\end{array}$ & Tüylüçay & $\begin{array}{l}\text { Çaye qwe, Çaye } \\
\text { çiya, Deme çole, } \\
\text { Deme bıri, Çayey } \\
\text { wariy, Dağ çayı, } \\
\text { Ada çayı, Dağ } \\
\text { çayı, Tokalı çay, } \\
\text { Tüylü çay, Bareş, } \\
\text { Tavsan kuyruğu, } \\
\text { Tüylü Kız }\end{array}$ & $\begin{array}{l}\text { Bingöl, Elazı̆̆, } \\
\text { Tunceli, Mersin, } \\
\text { Konya, Van, } \\
\text { Karaman, Erzincan }\end{array}$ & $\begin{array}{l}\text { Aerial parts, } \\
\text { flowering } \\
\text { branches, } \\
\text { inflorescence }\end{array}$ & $\begin{array}{l}\text { Tea (decoction, infusion } \\
\text { internally), powder }\end{array}$ & $\begin{array}{l}\text { Insomnia, colds, flu, sedative, food, } \\
\text { antipyretic, headache, stomach ache, } \\
\text { tonic, dsypnea healing (good for } \\
\text { shortness of breath), sore throat, } \\
\text { diabetes, urinary tract, bronchitis, } \\
\text { stomach ailments and respiratory tract, } \\
\text { animal diseases (for livestock), forage for } \\
\text { livestock (aerial parts) }\end{array}$ & $\begin{array}{l}\text { Yeşilada et al., 1993; } \\
\text { Tekin, 2011; Polat et } \\
\text { al., 2012; 2013; } \\
\text { Mükemre, 2013 } \\
\text { Alpaslan, 2012; Doğan, } \\
\text { 2014; Bağcı et al., } \\
\text { 2016; Olgun, } 2019\end{array}$ \\
\hline S. lavandulifolia var. & Tüylüçay & Tüylü Adaçayı & Gümüşhane & Aerial parts, & Tea, gargle & Fatigue and weakness, cough, stomach & Karakurt, 2014 \\
\hline
\end{tabular}




\begin{tabular}{|c|c|c|c|c|c|c|c|}
\hline $\begin{array}{l}\text { glabrescens } \\
\text { R.Bhattacharjee \& } \\
\text { Hub.-Mor. }\end{array}$ & & & & & & $\begin{array}{l}\text { ache, natural antibiotic, flu infections, } \\
\text { relaxing, to lower kidney stones, for body } \\
\text { resistance, nerves soothing effect, sore } \\
\text { throat, pharyngitis, bronchitis }\end{array}$ & \\
\hline $\begin{array}{l}\text { S. macrantha } \\
\text { (K.Koch) Stearn }\end{array}$ & Kocasoğulcan & $\begin{array}{l}\text { Karabaş, Koca } \\
\text { Soğulcan, Dağ } \\
\text { çayı }\end{array}$ & Rize, Trabzon & $\begin{array}{l}\text { Aerial parts, } \\
\text { inflorescence, } \\
\text { flowering } \\
\text { branches }\end{array}$ & Tea (infusion internally) & $\begin{array}{l}\text { Relaxing stomach (mixed with honey), } \\
\text { stimulant, anti-flatulent effect } \\
\text { (carminative), appetizer and stomachic, } \\
\text { anodyne }\end{array}$ & $\begin{array}{l}\text { Uzun and Uzun, 2011; } \\
\text { Saraç, 2013; } \\
\text { Saraç et al., } 2013\end{array}$ \\
\hline $\begin{array}{l}\text { S. mardinensis (Post) } \\
\text { R.R.Mill }\end{array}$ & Kayapungu & $\begin{array}{l}\text { Kaya Pungu } \\
\text { PungeTehta, } \\
\text { Rıhana tehtan, } \\
\text { Dağ çayı, Ot çayı, } \\
\text { Yayla çayı, Çeya } \\
\text { koyê, Çaya çiyan, } \\
\text { Birîngiya, } \\
\text { Giyapembik, } \\
\text { Punga teğtan, } \\
\text { Çaya çê, Mozik, } \\
\text { Ğev, Çaya beyanî }\end{array}$ & Şanlıurfa, Mardin & $\begin{array}{l}\text { Aerial parts } \\
\text { leaves }\end{array}$ & $\begin{array}{l}\text { Tea, aroma, as spices } \\
\text { (for 'tarhana'- a local } \\
\text { food) }\end{array}$ & $\begin{array}{l}\text { Aromatic, headache and diabetes } \\
\text { treatment cough, bronchitis disease }\end{array}$ & $\begin{array}{l}\text { Korkut, 2006; Akan et } \\
\text { al., 2008; Tuzlacı, } 2011 \\
\text { (as cited in Ertuğ, } \\
\text { 2014); Şeker, 2018; } \\
\text { Kılıç, } 2019\end{array}$ \\
\hline $\begin{array}{l}\text { S. megalodonta } \\
\text { subsp. mardinensis } \\
\text { R.Bhattacharjee* }\end{array}$ & Gevrekdeliçay & Rihana tehtan & Mardin & & Dried leaves as raw & In diabetes & Kılıç, 2019 \\
\hline $\begin{array}{l}\text { S. menthoides } \\
\text { Kotschy \& Boiss. }\end{array}$ & Nanedelisi & Rihana tehtan & Mardin & & Dried leaves as raw & In diabetes & Kılıç, 2019 \\
\hline $\begin{array}{l}\text { S. obliqua Waldst. \& } \\
\text { Kit. }\end{array}$ & Sarıçayçe & Dağ çayı & Balıkesir & $\begin{array}{l}\text { Aerial parts, } \\
\text { Flowering } \\
\text { branches }\end{array}$ & Tea (infusion) & Colds, flu, cough treatment, & $\begin{array}{l}\text { Polat, 2010; Polat and } \\
\text { Satı, 2012; Özdemir, } \\
2016\end{array}$ \\
\hline S. palustris L. & Gölısırganı & Oğul otu & Bursa, Rize & Aerial parts & $\begin{array}{l}\text { Powder of aerial parts of } \\
\text { the plant }\end{array}$ & For the reproduction of bees & $\begin{array}{l}\text { Aktan, 2011; Baykal et } \\
\text { al., } 2011\end{array}$ \\
\hline $\begin{array}{l}\text { S. pumila Banks \& } \\
\text { Sol. }\end{array}$ & Sarıkarabaş & Sarı karabaş & Şanlıurfa & & The dried plant is eaten & Stomach ache & Şeker, 2018 \\
\hline S. recta L. & Kara kurbağaotu & Dağçayı & Antalya & $\begin{array}{l}\text { Inflorescence, } \\
\text { leaves }\end{array}$ & Tea (infusion) & $\begin{array}{l}\text { Stimulant, anti-flatulent effect } \\
\text { (carminative), appetizer, stomach ache }\end{array}$ & $\begin{array}{l}\text { Baytop, 1994; 1999; } \\
\text { Özhatay et al., } 1997\end{array}$ \\
\hline $\begin{array}{l}\text { S. saturejoides } \\
\text { Montbret \& Aucher ex } \\
\text { Benth. }\end{array}$ & Çarşakdelisi & Çarşak delisi & Şanlıurfa & & Tea & & Şeker, 2018 \\
\hline $\begin{array}{l}\text { S. sericantha } \\
\text { P.H.Davis* }\end{array}$ & Dikenliçay & Dikenli çay & Antalya & Aerial parts & $\begin{array}{l}\text { Tea (infusion and } \\
\text { decoction) }\end{array}$ & Cold, stomach ailments, fever and cough & Kaya et al., 2017 \\
\hline $\begin{array}{l}\text { S. setifera subsp. lycia } \\
\text { (Gand.) } \\
\text { R.Bhattacharjee* }\end{array}$ & Zarifdeliçay & Zarif deliçay & Şanlıurfa & & Tea & & Şeker, 2018 \\
\hline $\begin{array}{l}\text { S. sosnowskyi } \\
\text { Kopell." }\end{array}$ & Oltudeliçayı & \multicolumn{4}{|c|}{ Original source is not specified. No usage details are given in the source. } & Medicinal & Koyu, 2020 \\
\hline
\end{tabular}




\begin{tabular}{|c|c|c|c|c|c|c|c|}
\hline $\begin{array}{l}\text { S. spectabilis Choisy } \\
\text { ex DC. }\end{array}$ & Alacakarabaş & Dağ çayı & Tunceli & Aerial parts & Tea (infusion) & Heart diseases & Doğan, 2014 \\
\hline S. sylvatica L. & Hamısırgan & $\begin{array}{l}\text { Turunç, Ham } \\
\text { sırgan }\end{array}$ & $\begin{array}{l}\text { Giresun, Rize, } \\
\text { Ankara }\end{array}$ & $\begin{array}{l}\text { Aerial parts, } \\
\text { leaves, flowering } \\
\text { branches }\end{array}$ & $\begin{array}{l}\text { Tea (infusion and } \\
\text { decoction) }\end{array}$ & Cardiac disorder, cough & $\begin{array}{l}\text { Simsek et al., 2004; } \\
\text { Baykal et al., 2011; } \\
\text { Polat et al., } 2015\end{array}$ \\
\hline S. thirkei K.Koch & Kestere & $\begin{array}{l}\text { Minare otu, } \\
\text { Tavşanak otu }\end{array}$ & Balıkesir & Aerial parts & Tea (infusion internally) & $\begin{array}{l}\text { Regulating blood pressure, colds, } \\
\text { digestive and carminative, relaxing the } \\
\text { nervous system }\end{array}$ & $\begin{array}{l}\text { Özdemir, 2016; Güner, } \\
2016 ; \text { Koyu, } 2020 \\
\text { (Original source is not } \\
\text { specified) }\end{array}$ \\
\hline S. tmolea Boiss. ${ }^{*}$ & Sürmeliçayçe & Kestire & Bilecik, Balıkesir & Aerial parts & Tea & Colds & Koyuncu et al., 2010 \\
\hline $\begin{array}{l}\text { S. viscosa Montbret \& } \\
\text { Aucher ex Benth. } \\
\text { (Syn: S. laetivirens) }^{* *}\end{array}$ & Yağlıkarabaş & $\begin{array}{l}\text { Ballıbaba, } \\
\text { Adaçayı, Çay otu, } \\
\text { Dağ çayı }\end{array}$ & $\begin{array}{l}\text { Gümüşhane, } \\
\text { Tunceli }\end{array}$ & $\begin{array}{l}\text { Inflorescence, } \\
\text { Aerial parts }\end{array}$ & $\begin{array}{l}\text { Nectar plant (for bees), } \\
\text { Tea (infusion) }\end{array}$ & Forage, colds, digestive & Karakurt, 2014 \\
\hline
\end{tabular}

* endemic, ${ }^{* \star}$ endemic before synonym 


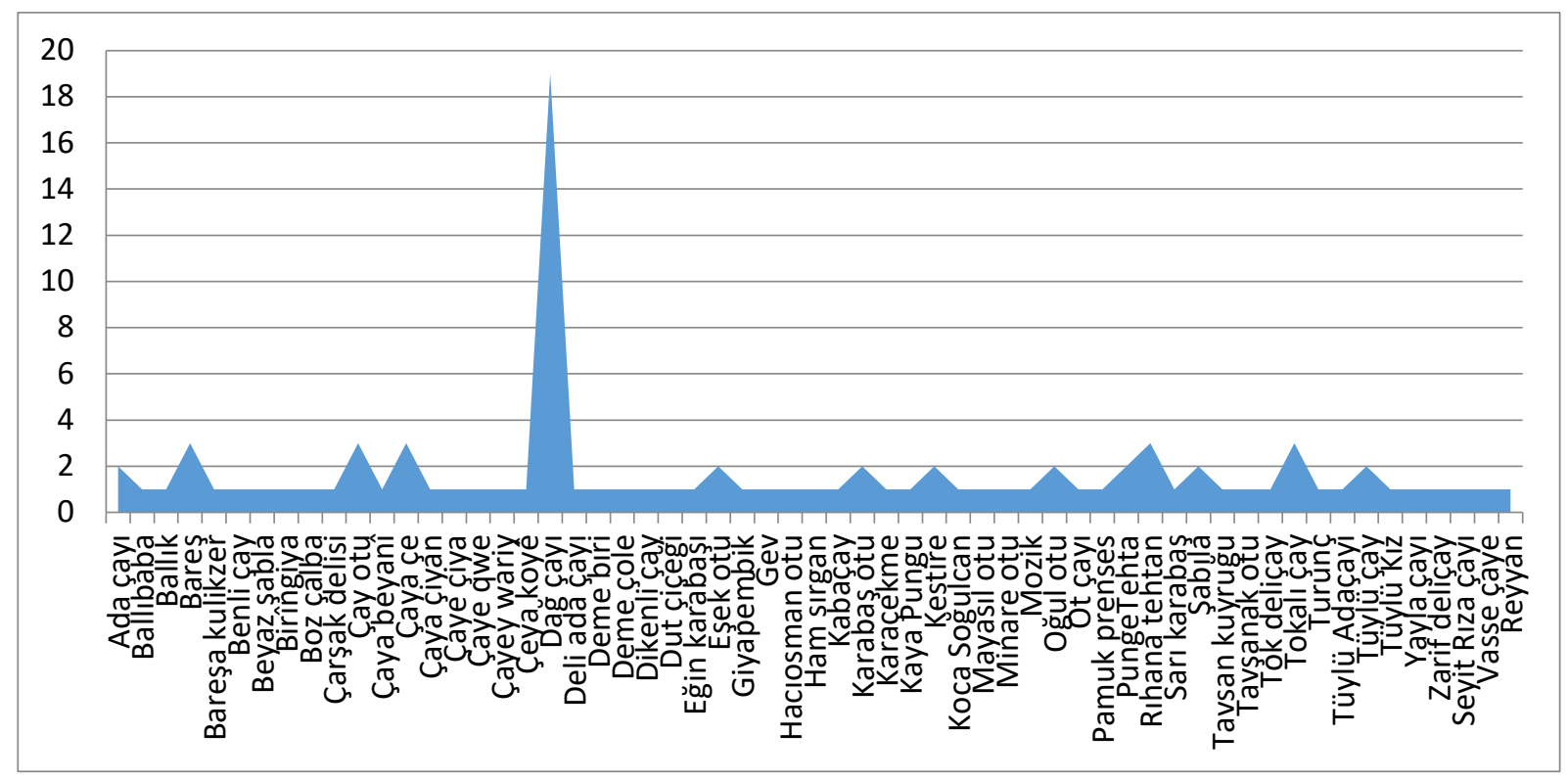

Figure 5. Vernacular names given to Stachys $L$. taxa (x axis: vernacular names, y axis: number of uses of names).

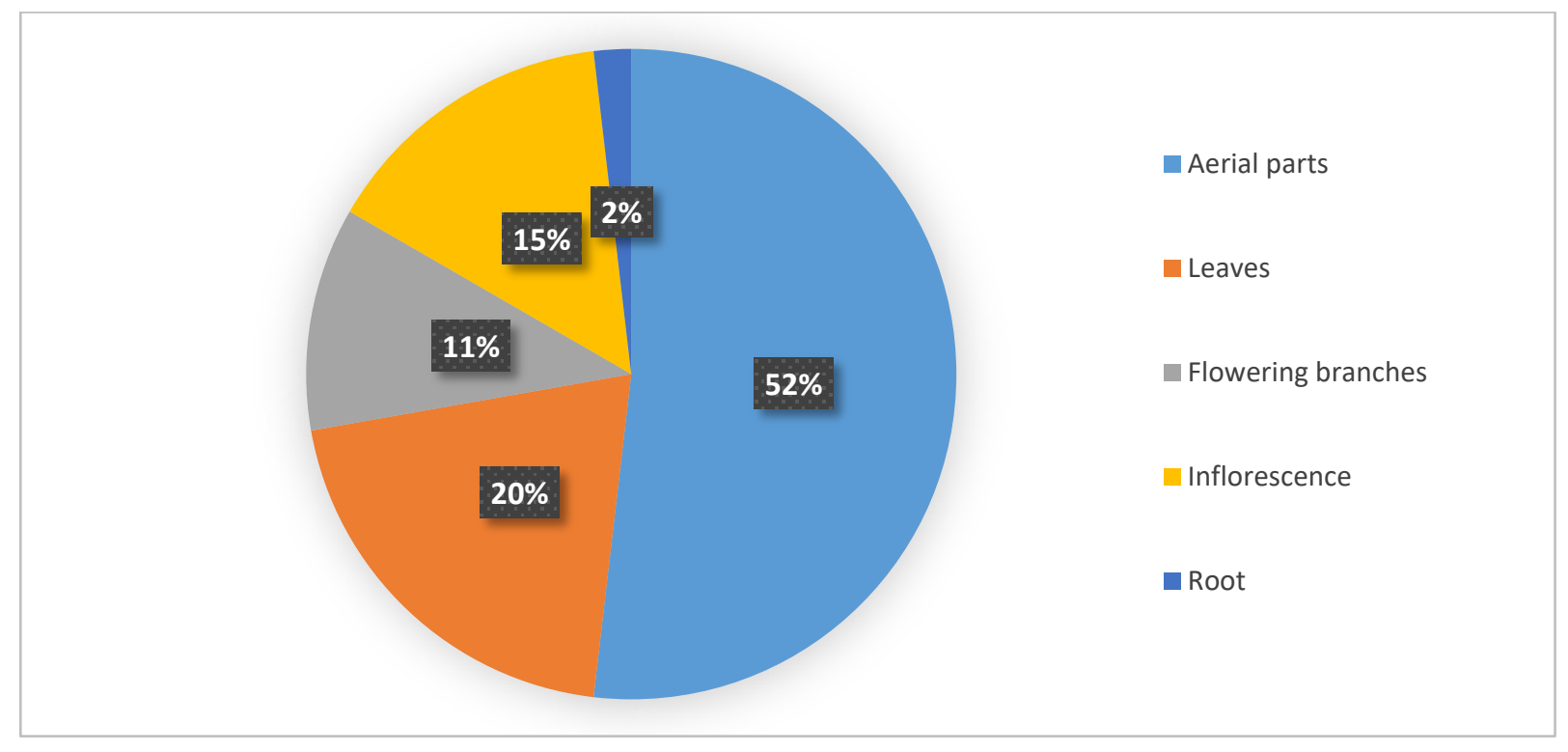

Figure 6. Parts of Stachys $L$. taxa used for ethnobotanical purposes. 


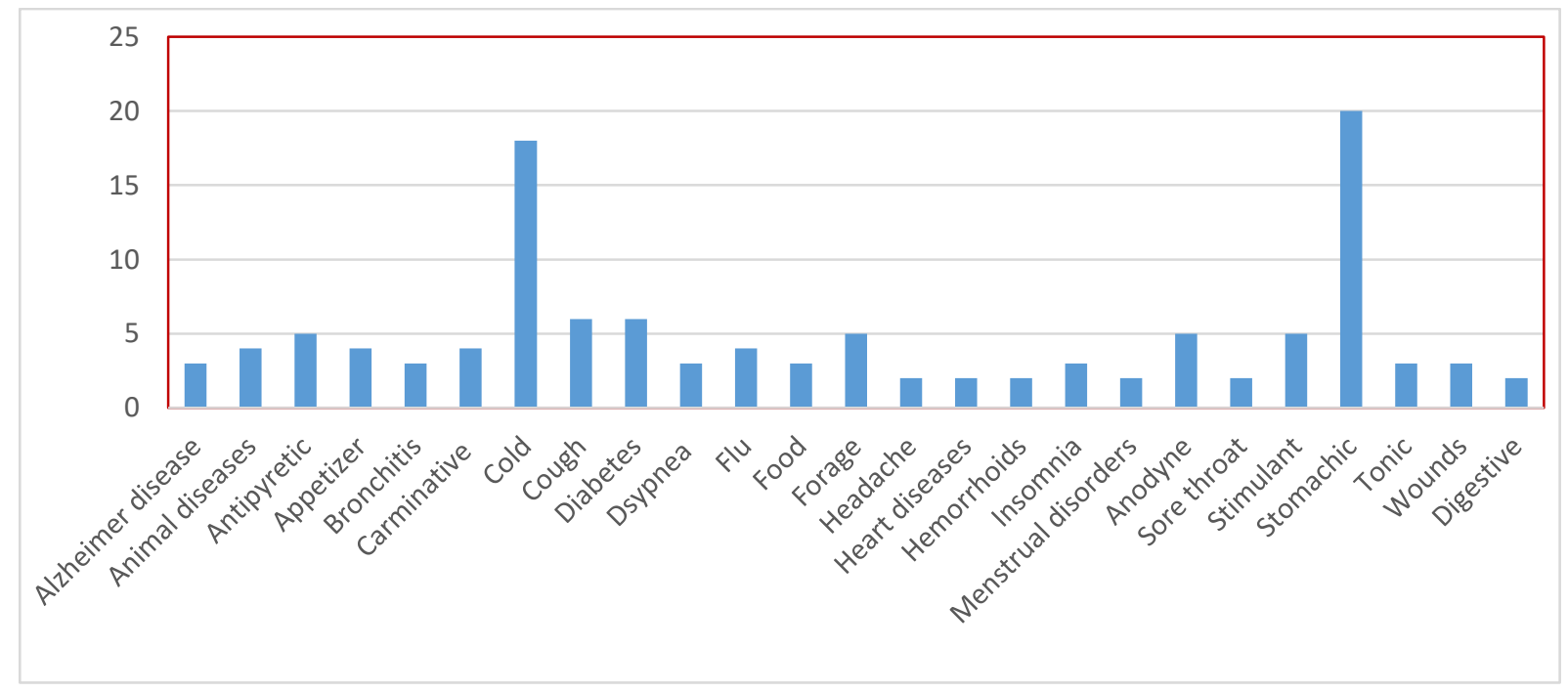

Figure 7. Purpose of use Stachys L. taxa according to ethnobotanical studies.

According to Goren (2014), many Stachys L. species are used in decoctions or infusions for the treatment of skin, stomach, ulcer, asthma, rheumatic disorders and vaginal tumours. However, in our study, the use of ulcers, asthma and vaginal tumours were not found.

The most common applications of $S$. lavandulifolia species are against fever, spasm, gastrodynia, dyspepsia, and flatulence. They also have sedative and anxiolytic effects (Goren, 2014). In our study, it was determined that many of these effects of this species are seen, as well as medical effects such as insomnia, euphoria effect, cough and other uses such as forage for livestock.

In Iran, the species S. germanica L. is a traditional medicine used in the treatment of painful menstruation and gastrodynia (Naghibi et al., 2005). However, although the mentioned species have spread in the Mediterranean region in our country, it has not been found any use among the people. In addition, S. lavandulifolia and S. balansae species are consumed as a tea in Azerbaijan (Goren, 2014). Although $S$. lavandulifolia is consumed as a tea in our country, $S$. balansae var. balansae has been used only in animal diseases in the form of powder. Its use in the form of tea has not been observed. In the world, Stachys officinalis (L.) Trevis. anti-infective on the scalp and skin (Di Sanzo et al., 2013). Lans et al. (2006) stated that it is effective in colic treatment. However, no use has been encountered in our country.

Stachys L. species are used as herbal medicine and generally consumed as a tea in Anatolia and Iran. It has known that "Dağ çayı (mountain tea)", infusions and decoctions of Stachys L. taxa are applied as tonics and stomach diseases, also antibacterial and antifungal effect (Ozturk et al., 2009). Inhibition of pain and inflammatory processes (Khanavi et al., 2005), anxiolytic effect (Rabbani et al., 2003), antibacterial (Grujic-Jovanovic et al., 2004), antinephritic agent (Hayashi et al., 1994), anticancer 
(Amirghofran et al., 2006), anti-helicobacter pylori (Stamatis et al., 2003), and antioxidant effects (Aydin et al., 2006) of explained in the literature. S. recta used as wound healing, another species, $S$. lavandulifolia is used for digestive disorders (Ozturk et al., 2009; Khanavi et al., 2009). However, although $S$. lavandulifolia has been used in digestive problems, it has not been found that $S$. recta is used for wound treatment.

Stachys palustris L. and Stachys sylvatica L. as the herb are used externally for the treatment of wounds and internally for abdominal pain, cramps, dizziness, fever, gout and menstrual disorders (in PDR for Herbal Medicines) (Gruenwald et al., 2000). However, in our study, such treatment was not observed in these species.

Besides, Stachys floridana Shuttlew. ex Benth., which is spread in China, is an important Chinese traditional plant for diabetics. In Anatolia, local people use plants to treat diabetes. Turkey was also seen in some of Stachys L. species is used in the treatment of diabetes (Cakilcioglu and Turkoglu, 2010; Xianfeng et al., 2013) (Table 1).

S. officinalis reported in Anthroposophic Pharmaceutical Codex (APC) (Jones et al., 2017). However, although $S$. officinalis has spread in our country, there is no record of use.

According to Haznagy-Radnai et al. (2008) S. officinalis, S. recta, S. sylvatica, and S. palustris are used as anti-inflammatory, anti-rheumatic, antibacterial drugs. In Hungarian folk medicine, these plants are also used as an antiphlogistic, spasmolytic, diuretic, sedative and for the treatment of tumour diseases. In addition, all of these species spread in our country. However, no use of S. officinalis species has been found. However, S. recta stimulant, anti-flatulent effect (carminative), appetizer, stomach ache; S. sylvatica cardiac disorder, cough and S. palustris are used for the purpose of the reproduction of bees, not medically. This shows that the same plants have revealed different usage patterns and purposes in different regions and cultures around the world.

Stachys L. taxa contain a wide variety of secondary metabolites. One of them is diterpenoids (Piozzi and Bruno, 2009). These diterpenoids have antibacterial, antifungal, antimycobacterial and antiAlzheimer activities (Goren, 2014). In our study, it was seen that there are Stachys L. species which are used in Alzheimer. These taxa are S. cretica subsp. garana, S. iberica subsp. iberica var. iberica, S. iberica subsp. stenostachya.

In his study, Goren (2014) stated that S. atherocalyx K.Koch was used as an anti-inflammatory in Iranian folk medicine. Also in Turkey, he said that they also used antibacterial purposes. However, such an application and data were not found in our researches.

S. iberica subsp. iberica var. iberica and S. cretica subsp. garana used for alzheimer in Anatolia. According to Bahadori et al. (2019) another S. cretica subspecies, S. cretica subsp. smyrnaea was evaluated for its antioxidant activity, phenolics profile, and therapeutic potential. Results showed 
that this plant contains amounts of significant phenolic acids and flavonoids. In addition, it was determined to be promising for anti-alzheimer, antidiabetic, antioxidant, and anti-tyrosinase potential. That study gives us $S$. cretica could be regarded as a new and rich source of bioactive ingredients for new formulations in cosmetics, functional foods, and pharmaceutical industries. This shows that the effects of herbs used in folk medicine in modern medicine are related.

Phytotherapy in animal diseases is applications from past to present. Therefore, such ethnoveterinary studies are important in the treatment and protection of animals. In the study, it has been seen that Stachys L. taxa have various uses for animals. The fresh aerial parts of the S. kurdica var. kurdica and S. balansae subsp. balansae are dried and pounded after drying and is used especially to treat the inflamed wounds of animals. In addition, S. lavandulifolia var. lavandulifolia is prepared in the same way and sprinkled on the wormy wounds of animals. Also, the infusion prepared from the aerial parts of $S$. cretica subsp. anatolica is drunk in rabies treatment for animals (internally).

Also, the aerial parts of Stachys L. taxa are generally used. However, it has been seen in the literature that only one study has root use. Its purpose is to use with the leaves for healing by rubbing them on the wounds (Güner, 2016).

\section{Conclusion}

The genus Stachys $L$. is represented by 118 taxa in our country. As a result of the researches, it is seen that approximately 1 in 3 of them have some kind of use (38 taxa, 13 of which are endemic).

The ethnobotanical use of genus is widely in the region covering from East to West Anatolia. However, no use has been identified in some regions of Turkey. According to ethnobotanical studies and the literature survey, Stachys L. species are generally consumed as herbal tea because of its volatile components (Such as Caryophyllene, germacrene D, a-pinene, linalool, $\beta$-pinene) and phenolic components.

The essential oil compositions of the Stachys L. genus have been well documented in the literature, but not for all the species have been described in detail.

Stachys L. taxa are used for most respiratory and stomach disorders in Turkey, as well as in diseases such as Alzheimer disease and diabetes were also found to be of use. With the idea that different people have similar uses of these species at different times and in different regions, we think that the examination of these herbal drugs will have a positive result for the treatment of related diseases.

At the same time, if Stachys L. taxa are not very popular like other known Lamiaceae plants (such as lavender, thyme, mint), There are many species of Stachys L. in Anatolia that are not yet known for any use. Stachys L. taxa would be more important in medicine by increasing their 
ethnobotanical studies and investigating the use of these plants in medicine and traditional medicine. Consequently, the Stachys L. taxa; It is one of the important potential plants that can be benefited in the preventive healthcare (especially as herbal tea), pharmaceutical and cosmetic (due to essential oils).

\section{Conflicts of Interests}

Authors declare that there is no conflict of interests

\section{References}

Akan, H., Korkut, M. M., Balos, M. M. (2008). An ethnobotanical study around Arat Mountain and its surroundings (Birecik, Sanlıurfa). FIrat University Journal of Science and Engineering, 20, $67-81$.

Akçiçek, E., Fırat, M., Güner, Ö. (2016). Stachys hakkariensis (Lamiaceae), a new species from Eastern Anatolia (Turkey) belonging to Stachys sect. Olisia. Phytotaxa, 257 (2), 167-173.

Aktan, T. (2011). Yenişehir (Bursa) Köylerinin Etnobotanik Özellikleri. Master Thesis, Manisa Celal Bayar University, Turkey.

Alpaslan, Z. (2012). Ergan Dağı (Erzincan)'nın Etnobotanik Özellikleri. Master Thesis, Erzincan Binali Yıldırım University, Turkey.

Altundag, E., Ozturk, M. (2011). Ethnomedicinal studies on the plant resources of east Anatolia, Turkey. Procedia-Social and Behavioral Sciences, 19, 756-777.

Amirghofran, Z., Bahmani, M., Azadmehr, A., Javidnia, K. (2006). Anticancer effects of various Iranian native medicinal plants on human tumor cell lines. Neoplasma, 53, 428-433.

Ayandın, H. (2010). Avşar, Şabanözü ve Çile Dağı (Polatı/Ankara) arasında kalan bölgenin etnobotanik özellikleri. PhD. Thesis, Selçuk University, Turkey.

Aydin, A., Sener, B., Cakici, I., Turan, N. N., Erdemoglu, N. (2006). Antioxidant activities of some Lamiaceae plant extracts. Phytotherapy Research, 20, 91-93.

Badem, M. (2017). Akkuş (Ordu) ve köylerinde bulunan bitkilerin etnobotanik ve etnomedikal özelliklerinin belirlenmesi ve halk ilacı olarak kullanılan türlerin biyolojik aktivitelerinin değerlendirilmesi. Master Thesis, Karadeniz Teknik University, Turkey.

Bağcl, Y. (2000). Ethnobotanical features of Aladaglar (Yahyalı, Kayseri) and its vicinity. The Herb Journal of Systematic Botany, 7, 89-94.

Bağcı, Y., Erdoğan, R., Doğu, S. (2016). Sarıveliler (Karaman) ve çevresinde yetişen bitkilerin etnobotanik özellikleri. Selçuk Üniversitesi Fen Fakültesi Fen Dergisi, 42 (1), 84-107. 
Bahadori, M. B., Kirkan, B., Sarikurkcu, C. (2019). Phenolic ingredients and therapeutic potential of Stachys cretica subsp. smyrnaea for the management of oxidative stress, Alzheimer's disease, hyperglycemia, and melasma. Industrial Crops and Products, 127, 82-87.

Balos, M. M., Akan, H. (2007). Zeytinbahçe-Akarçay (Birecik, Şanlıurfa) arasında kalan bölgenin etnobotanik özellikleri. Selçuk Üniversitesi Fen Fakültesi Fen Dergisi, 2 (29), 155-171.

Baykal, H. (2015). Başhemşin (Çamlıhemşin/Rize)'in florası, fitososyolojisi ve etnobotanik özellikleri. PhD. Thesis, Recep Tayyip Erdoğan University, Turkey.

Baykal, H., Yaldız, G., Yüksek, T. (2011). The medicinal and aromatic plants distrubiton of Rize flora. In 2nd International Non-Wood Products Symposium, 283.

Baytop, T. (1984). Türkiye' de Bitkilerle Tedavi, Geçmişte ve Bugün. İstanbul: İstanbul Üniversitesi Yayınları, No: $3255 / 40$.

Baytop, T. (1994). Türkçe Bitki Adları Sözlüğü (Vol. 578). Ankara: Türk Dil Kurumu.

Baytop, T. (1999). Türkiye’ de Bitkiler ile Tedavi (ilaveli ikinci baskı). İstanbul: Nobel Tıp Kitabevi, 193-194.

Bhattacharjee, R. (1980). Taxonomic studies in Stachys II: A new infrageneric classification of Stachys L.. Notes from the Royal Botanic Garden, Edinburgh, 38, 65-96.

Bulut, G. E. (2008). Bayramiç (Çanakkale) yöresinde etnobotanik araştırmalar. PhD. Thesis Marmara University, Turkey.

Cakilcioglu, U., Turkoglu, I. (2010). An ethnobotanical survey of medicinal plants in Sivrice (ElazığTurkey). Journal of Ethnopharmacology, 132 (1), 165-175.

Celep, F., Dirmenci, T. (2017). Systematic and Biogeographic overview of Lamiaceae in Turkey. Natural Volatiles and Essential Oils, 4 (4), 14-27.

Çömlekçioğlu, N., Karaman, Ş. (2008). Kahramanmaraş şehir merkezindeki aktarlarda bulunan tıbbi bitkiler. KSU Journal of Science and Engineering, 11 (1), 23-32.

Davis, P. H (Ed.). (1982). Flora of Turkey and the East Aegean Islands (Vol. 7), Edinburgh: Edinburgh University Press.

Di Sanzo, P., De Martino, L., Mancini, E., De Feo, V. (2013). Medicinal and useful plants in the tradition of Rotonda, Pollino National Park, Southern Italy. Journal of Ethnobiology and Ethnomedicine, 9 (1), 19.

Doğan, A. (2014). Pertek (Tunceli) yöresinde etnobotanik araştırmalar. PhD. Thesis, Marmara University, Turkey.

Dönmez, M., Kargıoğlu, M, Temel, M. (2012). Stachys palustris L.'in morfolojik, anatomik ve ekolojik Özellikleri. Afyon Kocatepe Üniversitesi Fen Bilimleri Dergisi, 12, 23-31. 
Duran, A. (1998). Akseki (Antalya) ilçesindeki bazı bitkilerin yerel adları ve etnobotanik özellikleri. Ot Sistematik Botanik Dergisi, 5 (1), 72-92.

Elçi, B., Erik, S. (2006). Güdül Ankara ve çevresinin etnobotanik özellikleri. Hacettepe Üniversitesi Eczacılık Fakültesi Dergisi, 2, 57-64.

Ertuğ, F. (2014). Etnobotanik Kaynakları. Resimli Türkiye Florası, 1.

Ertuğ, F. (2000). An ethnobotanical study in Central Anatolia (Turkey). Journal of Economic Botany, 54 (2), 155-182.

Ertuğ, F., Tümen, G., Çelik, A., Dirmenci, T. (2004). Buldan (Denizli) etnobotanik alan araştırması 2003. TÜBA Kültür Envanteri Dergisi, 2 (2), 187-218.

Everest, A., Ozturk, E. (2005). Focusing on the ethnobotanical uses of plants in Mersin and Adana provinces (Turkey). Journal of Ethnobiology and Ethnomedicine, 1 (1), 6.

Ezer, N., Avcı, K. (2004). Çerkeş (Çankırı) yöresinde kullanılan halk ilaçları. Hacettepe Üniversitesi Eczacılık Fakültesi Dergisi, 24, 67.

Ezer, N., Arisan, Ö. M. (2006). Folk medicines in Merzifon (Amasya, Turkey). Turkish Journal of Botany, 30 (3), 223-230.

Fakir, H., Korkmaz, M., Güller, B. (2009). Medicinal plant diversity of western Mediterrenean region in Turkey. Journal of Applied Biological Sciences, 3 (2), 33-43.

Goren, A. C., Akçiçek, E., Dirmenci, T., Kılıç T., Mozioğlu, E., Yılmaz, H. (2011a). Fatty acid composition and chemotaxonomic evaluation of species of Stachys. Natural Product Research, 26, 84-90.

Goren, A. C. Piozzi, F. Akçiçek E., Kılıç, T. Mozioğlu, E. Çarıkçı S., Seitzer W. N. (2011b). Essential oil composition of twenty-two Stachys species (mountain tea) and their biological activities. Phytochemistry Letters, 4, 448-453.

Goren, A. C. (2014). Use of Stachys species (Mountain Tea) as herbal tea and food. Records of Natural Products, 8 (2), 71-82.

Govaerts, R. (2003). World Checklist of Selected Plant Families Database in ACCESS: 1-216203. The Board of Trustees of the Royal Botanic Gardens, Kew (Access date: 22 October 2015).

Gruenwald, J., Brendler, T., Jaenicke, C., et al., (2000). PDR for Herbal Medicine. 2nd ed. Medical Economics Company, Montvale, NJ, p. 832.

Grujic-Jovanovic, Skaltsa, H. D., Marin, P., Sokovic, M. (2004). Composition and antimicrobial activity of the essential oil of six Stachys species from Serbia. Flavour And Fragrance Journal, 19, $139-144$.

Güldaş, N. (2009). Adıyaman ilinde etnobotanik değeri olan bazı bitkilerin kullanım alanlarının tespiti. Master Thesis, Firat University, Turkey. 
Guner, A., Ozhatay, N., Ekim, T., Baser, K. H. C. (2000). Flora of Turkey and the East Aegean Islands (Vol. 11), Edinburgh: Edinburgh University Press.

Güner, A., Aslan, S., Ekim, T., Vural, M., Babaç, M.T (Eds). (2012). Türkiye Bitkileri Listesi (Damarlı Bitkiler). İstanbul: Nezahat Gökyiğit Botanik Bahçesi ve Flora Araştırmaları Derneği.

Güner, Ö. (2016). Türkiye' de yetişen Stachys L. (Lamiaceae) cinsine ait Fragilicaulis R. Bhattacharjee seksiyonunun taksonomik revizyonu ve moleküler filogenetik analizi. PhD. Thesis, Balıkesir University, Turkey.

İlçim, A., Varol, Ö. (1996). Hatay ve Kahramanmaraş (Türkiye) illerindeki bazı bitkilerin etnobotanik özellikleri. Ot Sistematik Botanik Dergisi, 3 (1), 69-74.

Harley, R. M., Atkins, S., Budantsev, A., Cantino, P.D., Conn, B. J., Grayer, R. et al. (2004). Labiatae. In: Kadereit, J. W (Ed.), The Families and Genera of Vascular Plants. Berlin: Springer, 7, 167-275.

Hayashi, K., Nagamatsu, T., Ito, M., Hattori, T., Suzuki, Y. (1994). Acotoside, a component of Stachys sieboldii MIQ, may be a promising antinephritic agent. 1. Effects of acetoside on crescentic-type anti-GBM nephritis in rats. Japanese Journal of Pharmacology, 65, 143-151.

Haznagy-Radnai, E., Réthy, B., Czigle, S., Zupkó, I., Wéber, E., Martinek, T., Máthé, I. (2008). Cytotoxic activities of Stachys species. Fitoterapia, 79 (7-8), 595-597.

Işık, S., Gönüz, A., Arslan, Ü., Öztürk, M. (1995). Afyon (Türkiye) ilindeki bazı türlerin etnobotanik özellikleri. Ot Sistematik Botanik Dergisi, 2 (1), 161-166.

Jones, G., Maier, J., Pedersen, P., Schwarz, R., Mennet-von Eiff, M. (2017). Anthroposophic Pharmaceutical Codex APC. Dornach: International Association of Anthroposophic Pharmacists.

Karakurt, E. (2014). Kelkit (Gümüşhane) ilçesinin etnobotanik özellikleri. Master Thesis, Erzincan Binali Yıldırım University, Turkey.

Kaya, A., Demirci, B., Baser, K. H. C. (2001). The Composition of the essential oil of Stachys iberica subsp. stenostachya growing in Turkey. Chemistry of Natural Compounds, 37, 326-328.

Kaya, A., Demirci, B., Doğu, S., Dinç, M. (2017). Composition of the essential oil of Stachys sericantha, S. gaziantepensis, and S. mardinensis (Lamiaceae) from Turkey. International Journal of Food Properties, 20 (11), 2639-2644.

Keskin, L. (2011). Kadınhanı (Konya) ve çevresinde yetişen bitkilerin etnobotanik özellikleri. PhD. Thesis, Selçuk University, Turkey.

Khanavi, M., Sharifzadehb, M., Hadjiakhoondia, A., Shafieec, A. (2005). Phytochemical investigation and anti-inflammatory activity of aerial parts of Stachys byzanthina C. Koch. Journal of Ethnopharmacology, 97, 463-468. 
Khanavi, M., Hajimahmoodi, M., Cheraghi-Niroomand, M., Kargar, Z., Ajani, Y., Hadjiakhoondi, A., Oveisi, M. R. (2009). Comparison of the antioxidant activity and total phenolic contents in some Stachys species. African Journal of Biotechnology, 8 (6) 1143-1147.

Kılıç, M. (2019). Artuklu (Mardin) yöresinde yetişen bitkiler üzerine etnobotanik bir araştırma. PhD. Thesis, Manisa Celal Bayar University, Turkey.

Korkmaz, E., Demirkuş, N. (2019). Bahçesaray (Müküs/Van) ve çevresindeki gıda, yem, yakıt ve diğer amaçlar için kullanılan bitkilerin etnobotanik özellikleri. In: 8th Eurasian Conference on Language and Social Sciences, 78.

Korkut, M. M. (2006). Arat Dağı (Şanlıurfa) florası ve etnobotanik özellikleri. Master Thesis Harran University, Turkey.

Koyu, E. B. (2020). Türkiye' nin etnobotanik veritabanı. PhD. Thesis, Ege University, Turkey.

Koyuncu, O., Yaylaci, O., Öztürk, D., Potoglu Erkara, I., Savaroglu, F., Akcoskun, O., Ardic, M. (2010). Risk categories and ethnobotanical features of the Lamiaceae taxa growing naturally in Osmaneli (Bilecik/Turkey) and environs. Biological Diversity and Conservation, 3 (3), 31-45.

Lans, C., Turner, N., Brauer, G., Lourenco, G., Georges, K. (2006). Ethnoveterinary medicines used for horses in Trinidad and in British Columbia, Canada. Journal of Ethnobiology and Ethnomedicine, 2 (1), 31.

Maleki, N., Garjan,i, A., Nazemiyeh, H., Nilfouroushan, N., Eftekhar Sadat, A.T., Allameh, Z., Hasannia, N. (2001). Potent anti-inflammatory activities of hydroalcoholic extract from aerial parts of Stachys inflata on rats. Journal of Ethnopharmacology, 75, 213-218.

Metin, A. (2009). Mut ve çevresinde yetişen bitkilerin (Mersin) etnobotanik özellikleri. Master Thesis, Selçuk University, Turkey.

Muthu, C., Ayyanar, M., Raja, N., Ignacimuthu, S. (2006). Medicinal plants used by traditional healers in Kancheepuram district of Tamil Nadu, India. Journal of Ethnobiology and Ethnomedicine, 2, 43.

Mükemre, M. (2013). Konalga, Sırmalı, Dokuzdam köyleri (Çatak-Van) ve çevrelerinin etnobotanik özellikleri. Master Thesis, Yüzüncü Yıl University, Turkey.

Naghibi, F., Mosaddegh, M., Motamed, S.M., Ghorbani, A. (2005). Labiatae family in folk medicine in Iran: from ethnobotany to pharmacology. Iranian Journal of Pharmaceutical Research, 2, 6379.

Olgun, Ş. (2019). Arıcak (Elazığ) ilçesinin etnobotanik özellikleri. Master Thesis, Bingöl University, Turkey. 
Ozturk, M., Duru, M. E., Aydogmus-Ozturk, F., Harmandar, M., Mahlicli, M., Kolak, U., Ulubelen, A. (2009). GC-MS analysis and antimicrobial activity of essential oil of Stachys cretica subsp. smyrnaea. Natural Product Communications, 4 (1), 109-114.

Özdemir Nath, E. (2016). An ethnobotanical study in Savaştepe and Kepsut region (Balıkesir). $\mathrm{PhD}$. Thesis, Istanbul University, Turkey.

Özgen, U., Kaya, Y., Coşkun, M. (2004). Ethnobotanical studies in the villages of Ilıca (ErzurumTurkey) district. Economic Botany, 58 (4), 691-696.

Özhatay, N., Koyuncu, M., Atay, S., Byfield, A. (1997). Türkiye'nin Doğal Tıbbi Bitkilerinin Ticareti Hakkında Bir Çalışma. İstanbul: Doğal Hayatı Koruma Derneği Yayınları, No: 38-39.

Piozzi F., Bruno M. (2009). Diterpenoids in the essential oils from the genus Stachys. Records of Natural Products, 3, 120-125.

Polat, R. (2010). Havran ve Burhaniye (Balıkesir) çevresinde tarımsal biyoçeşitlilik ve etnobotanik araştırmaları. PhD. Thesis, Balıkesir University, Turkey.

Polat, R., Satıl, F. (2012). An ethnobotanical survey of medicinal plants in Edremit Gulf (BalıkesirTurkey). Journal of Ethnopharmacology, 139 (2), 626-641.

Polat, R., Cakilcioglu, U., Satı, F. (2013). Traditional uses of medicinal plants in Solhan (BingölTurkey). Journal of Ethnopharmacology, 148 (3), 951-963.

Polat, R., Cakilcioglu, U., Kaltalioğlu, K., Ulusan, M. D., Türkmen, Z. (2015). An ethnobotanical study on medicinal plants in Espiye and its surrounding (Giresun-Turkey). Journal of Ethnopharmacology, 163, 1-11.

Polat, R., Selvi, S., Cakilcioglu, U., Acar, M. (2012). Investigations of ethnobotanical aspect of wild plants sold in Bingöl (Turkey) local markets. Biological Diversity and Conservation, 5 (3), 155161.

Rabbani, M., Sajjadi, S. E., Zarei, H. R. (2003). Anxiolytic effects of Stachys lavandulifolia Vahl on the elevated plus-maze model of anxiety in mice. Journal of Ethnopharmacology, 89, 271-276.

Saraç, D. U. (2013). Rize ili etnobotanik özellikleri. Master Thesis, Karadeniz Teknik University, Turkey.

Saraç, D. U., Özkan, Z. C., Akbulut, S. (2013). Ethnobotanic features of Rize/Turkey province. Biological Diversity and Conservation, 6 (3), 57-66.

Sezik, E., Tabata, M., Yeşilada, E., Honda, G., Goto, K., Ikeshiro, Y. (1991). Traditional medicine in Turkey I. Folk medicine in North-East Anatolia. Journal of Ethnopharmacology, 35,191-196.

Sezik, E., Yeşilada, E., Tabata, M., Honda, G., Takaishi, Y., Fujita, T., Tanaka, T., Takeda, Y. (1997). Traditional medicine in Turkey VIII. Folk medicine in East Anatolia; Erzurum, Erzincan, Ağrı, Iğdır Provinces. Journal of Economic Botany, 51 (3), 195-211. 
Simsek, I., Aytekin, F., Yesilada, E., Yildirimli, Ş. (2004). An ethnobotanical survey of the Beypazari, Ayas, and Güdül district towns of Ankara Province (Turkey). Economic Botany, 58 (4), 705.

Stamatis, G., Kyriazopoulos, P., Golegou, S., Basayiannis, A., Skaltsas, S., Skaltsa, H. (2003). In vitro anti-Helicobacter pylori activity of Grek herbal medicines. Journal of Ethnopharmacology, $88,175-179$.

Şeker, F. (2018). Şanlıurfa ili Lamiaceae (Ballıbabagiller) familyasının florası bazı taksonların fitokimyasal ve etnobotanik özellikleri. PhD. Thesis, Recep Tayyip Erdoğan University, Turkey.

Şenkardeş, İ. (2014). Nevşehir'in güney ilçelerinde (Acıgöl, Derinkuyu, Gülşehir, Nevşehir-Merkez, Ürgüp) etnobotanik araştırmalar. PhD. Thesis, Marmara University, Turkey.

Takeda Y., Zhang, H., Masuda, T., Honda, G., Otsuka, H., Sezik, E., Yeşilada, E., Sun, H. (1996). Megastigmane glucosides from Stachys byzantina. Phytochemistry, 44 (7), 1335-1337.

Tekin, S. (2011). Üzümlü (Erzincan) ilçesinin etnobotanik özellikleri. Master Thesis, Erzincan Binali Yıldırım University, Turkey.

Uzun, A., Palabaş, Uzun, S. (2011). Medicinal and aromatic plant taxa of Altındere valley (Maçka/Trabzon) 2nd International Non-Wood Forest Products Symposium 8-10 September 2011- Isparta/Turkey, 267-282.

Vural, G. (2008). Honaz dağı ve çevresindeki bazı doğal bitkilerin etnobotanik özellikleri. Master Thesis, Afyon Kocatepe University, Turkey.

Xianfeng, Z., Guidong, H., Yan, C. et al. (2013). Optimization of extracting stachyose from Stachys floridana Schuttl. ex Benth by response surface methodology. Journal of Food Science and Technology, 50, 942-949.

Yeşil, Y. (2007). Kürecik (Akçadağ/Malatya) bucağında etnobotanik bir araştırma. Master Thesis, İstanbul University, Turkey.

Yeşil, Y., Akalın, E. (2009). Folk medicinal plants in Kürecik area (Akçadağ/Malatya Turkey). Turkish Journal of Pharmaceutical Sciences, 6 (3), 207-220.

Yeşilada, E., Honda, G., Sezik, E., Tabata, M., Fujita, T., Tanaka, T., Takaishi, Y. (1995). Traditional medicine in Turkey. V. Folk medicine in the inner Taurus Mountains. Journal of Ethnopharmacology, 46 (3), 133-152.

Yeşilada, E., Honda, G., Sezik, E., Tabata, M., Goto, K., Ikeshiro, Y. (1993). Traditional medicine in Turkey IV. folk medicine in the Mediterranean subdivision. Journal of Ethnopharmacology, 39 (1), 31-38. 